\title{
Multivariable Feedback Design: Concepts for a Classical/Modern Synthesis
}

\author{
JOHN C. DOYLE AND GUNTER STEIN, MEMBER, IEEE
}

\begin{abstract}
This paper presents a practical design perspective on multivariable feedback control problems. It reviews the basic issue-feedback design in the face of uncertaintics-and generalizes known single-input, single-output (SISO) statements and constraints of the design problem to multiinput, multioutput (MIMO) cases. Two major MIMO design approaches are then evaluated in the context of these results.
\end{abstract}

\section{INTRODUCTION}

$\mathrm{T}$ HE last two decades have brought major developments in the mathematical theory of multivariable linear time invariant feedback systems. These include the celebrated state space concept for system description and the notions of mathematical optimization for controller synthesis [1], [2]. Various time-domain-based analytical and computational tools have been made possible by these ideas. The developments also include certain generalizations of frequency-domain concepts which offer analysis and synthesis tools in the classical single-input, singleoutput (SISO) tradition [3], [4]. Unfortunately, however, the two decades have also brought a growing schism between practitioners of feedback control design and its theoreticians. The theory has increasingly concentrated on analytical issues and has placed little emphasis on issues which are important and interesting from the perspective of design.

This paper is an attempt to express the latter perspective and to examine the extent to which modern results are meaningful to it. The paper begins with a review of the fundamental practical issue in feedback design-namely, how to achieve the benefits of feedback in the face of uncertainties. Various types of uncertainties which arise in physical systems are briefly described and so-called "unstructured uncertainties" are singled out as generic errors which are associated with all design models. The paper then shows how classical SISO statements of the feedback design problem in the face of unstructured uncertainties can be reliably generalized to multiinput, multioutput (MIMO) systems, and it develops MIMO generalizations

Manuscript received March 13, 1980; revised October 6, 1980. This research was supported by the ONR under Contract N00014-75-C-0144, by the DOE under Contract ET-78-C-01-3391, and by NASA under Grant NGL-22-009-124.

J. C. Doyle is with the Systems and Research Center, Honeywell, Inc. Minneapolis, MN 55413 and the University of California, Berkeley, CA 94720.

G. Stein is with the Systems and Research Center, Honeywell, Inc. Minneapolis, MN 55413 and the Department of Electrical Engineering and Computer Sciences, Massachusetts Institute of Technology, Cambridge, MA 02139 . of the classical Bode gain/phase constraints [5], [6] which limit ultimate performance of feedback in the face of such uncertainties. Several proposed MIMO design procedures are examined next in the context of the fundamental feedback design issue. These include the recent frequency domain inverse Nyquist array (INA) and characteristic loci (CL) methods and the well-known linear-quadratic Gaussian (LQG) procedure. The INA and CL methods are found to be effective, but only in special cases, while LQG methods, if used properly, have desirable general features. The latter are fortunate consequences of quadratic optimization, not explicitly sought after or tested for by the theoretical developers of the procedure. Practitioners should find them valuable for design.

\section{Feedback Fundamentals}

We will deal with the standard feedback configuration illustrated in Fig. 1. It consists of the interconnected plant $(G)$ and controller $(K)$ forced by commands $(r)$, measurement noise $(\eta)$, and disturbances $(d)$. The dashed precompensator $(P)$ is an optional element used to achieve deliberate command shaping or to represent a nonunity feedback system in equivalent unity feedback form. All disturbances are assumed to be reflected to the measured outputs $(y)$, all signals are multivariable, in general, and both nominal mathematical models for $G$ and $K$ are finite dimensional linear time invariant (FDLTI) systems with transfer function matrices $G(s)$ and $K(s)$. Then it is well known that the configuration, if it is stable, has the following major properties:

1) Input-Output Behavior:

$$
\begin{aligned}
y & =G K(I+G K)^{-1}(r-\eta)+(I+G K)^{-1} d \\
e & \triangleq r-y \\
& =(I+G K)^{-1}(r-d)+G K(I+G K)^{-1} \eta .
\end{aligned}
$$

2) System Sensitivity [7]:

$$
\Delta H_{\mathrm{cl}}=\left(I+G^{\prime} K\right)^{-1} \Delta H_{\mathrm{ol}} \text {. }
$$

In (3), $\Delta H_{\mathrm{cl}}$ and $\Delta H_{\mathrm{ol}}$ denote changes in the closed-loop system and changes in a nominally equivalent open-loop system, respectively, caused by changes in the plant $G$, i.e., $G^{\prime}=G+\Delta G$.

Equations (1)-(3) summarize the fundamental benefits and design objectives inherent in feedback loops. Specifically, (2) shows that the loop's errors in the presence of 


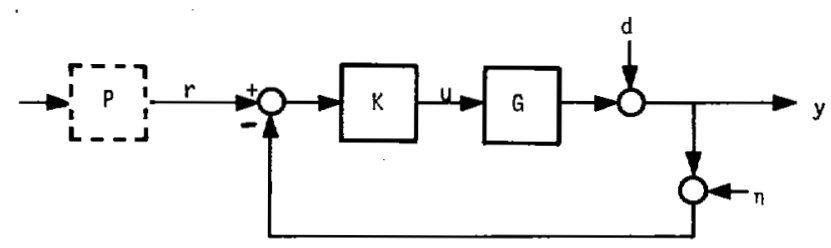

Fig. 1. Standard feedback configuration.

commands and disturbances can be made "small" by making the sensitivity operator, or inverse return difference operator, $(I+G K)^{-1}$, "small," and (3) shows that loop sensitivity is improved under these same conditions, provided $G^{\prime}$ does not stray too far from $G$.

For SISO systems, the appropriate notion of smallness for the sensitivity operator is well-understood-namely, we require that the complex scalar $[1+g(j \omega) k(j \omega)]^{-1}$ have small magnitude, or conversely that $1+g(j \omega) k(j \omega)$ have large magnitude, for all real frequencies $\omega$ where the commands, disturbances and/or plant changes, $\Delta G$, are significant. In fact, the performance objectives of SISO feedback systems are commonly stipulated in terms of explicit inequalities of the form

$$
p s(\omega) \leqslant|1+g(j \omega) k(j \omega)| \quad \forall \omega \leqslant \omega_{0},
$$

where $p s(\omega)$ is a (large) positive function and $\omega_{0}$ specifies the active frequency range.

This basic idea can be readily extended to MIMO problems through the use of matrix norms. Selecting the spectral norms as our measure of matrix size, for example, the corresponding feedback requirements become

$$
\bar{\sigma}\left[(I+G(j \omega) K(j \omega))^{-1}\right] \quad \text { small }
$$

or conversely

$$
p s(\omega) \leqslant \underline{\sigma}[I+G(j \omega) K(j \omega)]
$$

for the necessary range of frequencies. The symbols $\bar{\sigma}$ and $\underline{\sigma}$ in these expressions are defined as follows:

$$
\begin{aligned}
& \bar{\sigma}[A] \triangleq \max _{\|x\|=1}\|A x\| \equiv \sqrt{\lambda_{\max }\left[A^{*} A\right]} \\
& \underline{\sigma}[A] \triangleq \min _{\|x\|=1}\|A x\| \equiv \sqrt{\lambda_{\min }\left[A^{*} A\right]}
\end{aligned}
$$

where $\|\cdot\|$ is the usual Euclidean norm, $\lambda[\cdot]$ denotes eigenvalues, and $[\cdot]^{*}$ denotes conjugate transpose. The two $\sigma$ 's are called maximum and minimum singular values of $A$ (or principal gains [4]), respectively, and can be calculated with available linear system software [8]. More discussion of singular values and their properties can be found in various texts [9].

Condition (5) on the return difference $I+G K$ can be interpreted as merely a restatement of the common intuition that large loop gains or "tight" loops yield good performance. This follows from the inequalities

$$
\underline{\sigma}[G K]-1 \leqslant \underline{\sigma}[I+G K] \leqslant \underline{\sigma}[G K]+1
$$

which show that return difference magnitudes approximate the loop gains, $\underline{\sigma}[G K]$, whenever these are large compared with unity. Evidently, good multivariable feedback loop design boils down to achieving high loop gains in the necessary frequency range.

Despite the simplicity of this last statement, it is clear from years of research and design activity that feedback design is not trivial. This is true because loop gains cannot be made arbitrarily high over arbitrarily large frequency ranges. Rather, they must satisfy certain performance tradeoffs and design limitations. A major performance tradeoff, for example, concerns command and disturbance error reduction versus sensor noise error reduction [10]. The conflict between these two objectives is evident in (2). Large $\boldsymbol{\sigma}[G K(j \omega)]$ values over a large frequency range make errors due to $r$ and $d$ small. However, they also make errors due to $\eta$ large because this noise is "passed through" over the same frequency range, i.e.,

$$
y=G K(j \omega)[I+G K(j \omega)]^{-1} \eta \approx I \eta .
$$

Worse still, large loop gains can make the control activity (variable $u$ in Fig. 1) quite unacceptable. This follows from

$$
\begin{aligned}
u & =K[I+G K]^{-1}(r-\eta-d) \\
& \approx G^{-1}(j \omega)(r-\eta-d) .
\end{aligned}
$$

Here we have assumed $G$ to be square and invertible for convenience. The resulting equation shows that commands, disturbances, and sensor noise are actually amplified at $u$ whenever the frequency range significantly exceeds the bandwidth of $G$, i.e., for $\omega$ such that $\bar{\sigma}[G(j \omega)] \ll 1$ we get

$$
\underline{\sigma}\left[G^{-1}(j \omega)\right] \equiv \frac{1}{\bar{\sigma}[G(j \omega)]} \gg 1
$$

One of the major contributions of modern feedback theory is the development of systematic procedures for conducting the above performance tradeoffs. We are referring, of course, to the LQG theory [11] and to its modern Wiener-Hopf frequency domain counterpart [12]. Under reasonable assumptions on plant, disturbances, and performance criteria, these procedures yield efficient design compromises. In fact, if the tradeoff between command/disturbance error reduction and sensor noise error reduction were the only constraint on feedback design, practitioners would have little to complain about with respect to the relevance of modern theory. The problem is that these performance trades are often overshadowed by a second limitation on high loop gainsnamely, the requirement for tolerance to uncertainties. Although a controller may be designed using FDLTI models, the design must be implemented and operate with a real physical plant. The properties of physical systems, in particular the ways in which they deviate from finitedimensional linear models, put strict limitations on the 
frequency range over which the loop gains may be large. In order to properly motivate these restrictions, we digress in Section III to a brief description of the types of system uncertainties most frequently encountered. The manner in which these uncertainties can be accounted for in MIMO design then forms the basis for the rest of the paper.

\section{UNCERTAINTIES}

While no nominal design model $G(s)$ can emulate a physical plant perfectly, it is clear that some models do so with greater fidelity than others. Hence, no nominal model should be considered complete without some assessment of its errors. We will call these errors the "model uncertainties," and whatever mechanism is used to express them will be called a "representation of uncertainty."

Representations of uncertainty vary primarily in terms of the amount of structure they contain. This reflects both our knowledge of the physical mechanisms which cause differences between model and plant and our ability to represent these mechanisms in a way that facilitates convenient manipulation. For example, a set membership statement for the parameters of an otherwise known FDLTI model is a highly structured representation of uncertainty. It typically arises from the use of linear incremental models at various operating points, e.g., aerodynamic coefficients in flight control vary with flight environment and aircraft configurations, and equation coefficients in power plant control vary with aging, slag buildup, coal composition, etc. In each case, the amounts of variation and any known relationships between parameters can be expressed by confining the parameters to appropriately defined subsets of parameter space. A specific example of such a parameterization for the F-8C aircraft is given in [13]. Examples of less-structured representations of uncertainty are direct set membership statements for the transfer function matrix of the model. For instance, the statement

$$
G^{\prime}(j \omega)=G(j \omega)+\Delta G(j \omega)
$$

with

$$
\bar{\sigma}[\Delta G(j \omega)]<l_{a}(\omega) \quad \forall \omega \geqslant 0
$$

where $l_{a}(\cdot)$ is a positive scalar function, confines the matrix $G^{\prime}$ to a neighborhood of $G$ with magnitude $l_{a}(\omega)$. The statement does not imply a mechanism or structure which gives rise to $\Delta G$. The uncertainty may be caused by parameter changes, as above, or by neglected dynamics, or by a host of other unspecified effects. An alternative statement for (12) is the so-called multiplicative form:

$$
G^{\prime}(j \omega)=[I+L(j \omega)] G(j \omega)
$$

with

$$
\bar{\sigma}[L(j \omega)]<l_{m}(\omega) \quad \forall \omega \geqslant 0 .
$$

This statement confines $G^{\prime}$ to a normalized neighborhood of $G$. It is preferable over (12) because compensated transfer functions have the same uncertainty representation as the raw model (i.e., the bound (13) applies to $G K$ as well as to $G$ ). Still other alternative set membership statements are the inverse forms of (12) and (13) which confine $\left(G^{\prime}\right)^{-1}$ to direct or normalized neighborhoods about $G^{-1}$.

The best choice of uncertainty representation for a specific FDLTI model depends, of course, on the errors the model makes. In practice, it is generally possible to represent some of these errors in a highly structured parameterized form. These are usually the low frequency error components. There are always remaining higher frequency errors, however, which cannot be covered this way. These are caused by such effects as infinitedimensional electromechanical resonances [16], [17], time delays, diffusion processes, etc. Fortunately, the lessstructured representations, (12) or (13), are well suited to represent this latter class of errors. Consequently, (12) and (13) have become widely used "generic" uncertainty representations for FDLTI models.

Motivated by these observations, we will focus throughout the rest of this paper exclusively on the effects of uncertainties as represented by (13). For lack of a better name, we will refer to these uncertainties simply as "unstructured." We will assume that $G^{\prime}$ in (13) remains a strictly proper FDLTI system and that $G^{\prime}$ has the same number of unstable modes as $G$. The unstable modes of $G^{\prime}$ and $G$ do not need to be identical, however, and hence $L(s)$ may be an unstable operator. These restricted assumptions on $G^{\prime}$ make exposition easy. More general perturbations (e.g., time varying, infinite dimensional, nonlinear) can also be covered by the bounds in (13) provided they are given appropriate "conic sector" interpretations via Parseval's theorem. This connection is developed in [14], [15] and will not be pursued here.

When used to represent the various high frequency mechanisms mentioned above, the bounding functions $l_{m}(\omega)$ in (13) commonly have the properties illustrated in Fig. 2. They are small $(\ll 1)$ at low frequencies and increase to unity and above at higher frequencies. The growth with frequency inevitably occurs because phase uncertainties eventually exceed \pm 180 degrees and magnitude deviations eventually exceed the nominal transfer function magnitudes. Readers who are skeptical about this reality are encouraged to try a few experiments with physical devices.

It should also be noted that the representation of uncertainty in (13) can be used to include perturbation effects that are in fact not at all uncertain. A nonlinear element, for example, may be quite accurately modeled, but because our design techniques cannot deal with the nonlinearity effectively, it is treated as a conic linearity [14], [15]. As another example, we may deliberately choose to ignore various known dynamic characteristics in order to achieve a simpler nominal design model. 

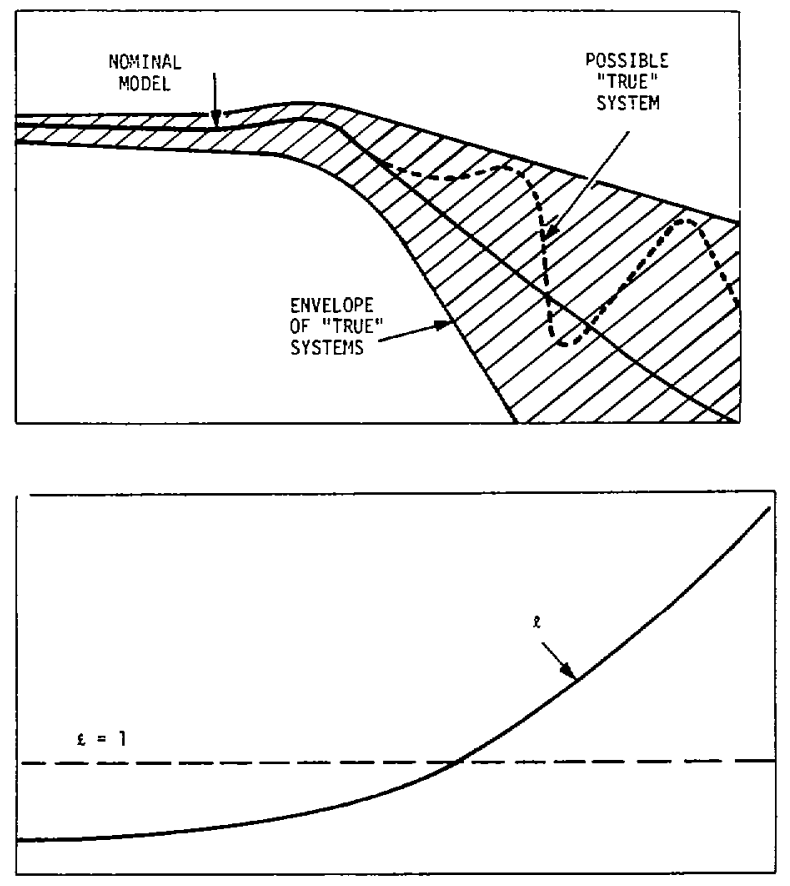

LOG FREOUENCY

Fig. 2. Typical behavior of multiplicative perturbations.

Another important point is that the construction of $l_{m}(\omega)$ for multivariable systems is not trivial. The bound assumes a single worst case uncertainty magnitude applicable to all channels. If substantially different levels of uncertainty exist in various channels, it may be necessary to scale the input-output variables and/or apply frequency-dependent transformations [15] in such a way that $l_{m}$ becomes more uniformly tight. These scale factors and transformations are here assumed to be part of the nominal model $G(s)$.

\section{Feedback Design in the Face of UNSTRUCTURED UNCERTAINTIES}

Once we specify a design model, $G(s)$, and accept the existence of unstructured uncertainties in the form (13), the feedback design problem becomes one of finding a compensator $K(s)$ such that

1) the nominal feedback system, $G K[I+G K]^{-1}$, is stable;

2) the perturbed system, $G^{\prime} K\left[I+G^{\prime} K\right]^{-1}$, is stable for all possible $G^{\prime}$ allowed by (13); and

3) performance objectives are satisfied for all possible $G^{\prime}$ allowed by (13).

All three of these requirements can be interpreted as frequency domain conditions on the nominal loop transfer matrix, $G K(s)$, which the designer must attempt to satisfy.

\section{Stability Conditions}

The frequency domain conditions for requirement 1) are, of course, well known. In SISO cases, they take the form of the standard Nyquist criterion, ${ }^{1}$ and in MIMO cases, they involve its multivariable generalization [18]. Namely, we require that the encirclement count of the map $\operatorname{det}[I+G K(s)]$, evaluated on the standard Nyquist $D$-contour, be equal to the (negative) number of unstable open loop modes of $G K$.

Similarly, for requirement 2) the number of encirclements of the map $\operatorname{det}\left[I+G^{\prime} K(s)\right]$ must equal the (negative) number of unstable modes of $G^{\prime} K$. Under our assumptions on $G^{\prime}$, however, this number is the same as that of $G K$. Hence, requirement 2) is satisfied if and only if the number of encirclements of $\operatorname{det}\left[I+G^{\prime} K(s)\right]$ remains unchanged for all $G^{\prime}$ allowed by (13). This is assured iff $\operatorname{det}\left[I+G^{\prime} K\right]$ remains nonzero as $G$ is warped continuously toward $G^{\prime}$, or equivalently, iff

$$
0<\underline{\sigma}[I+[I+\epsilon L(\bar{s})] G(\bar{s}) K(\bar{s})]
$$

for all $0 \leqslant \epsilon \leqslant 1$, all $\bar{s}$ on the $D$-contour, and all $L(s)$ satisfying (13). Since $G^{\prime}$ vanishes on the infinite radius segment of the $D$-contour, and assuming, for simplicity, that the contour requires no indentations along the $j \omega$ axis, $^{2}(14)$ reduces to the following equivalent conditions:

$$
0<\underline{\sigma}[I+G(j \omega) K(j \omega)+\epsilon L(j \omega) G(j \omega) K(j \omega)]
$$

for all $0 \leqslant \epsilon \leqslant 1,0 \leqslant \omega<\infty$, and all $L$.

$$
\Leftrightarrow 0<\underline{\underline{g}}\left[I+L G K(I+G K)^{-1}\right]
$$

for all $0 \leqslant \omega<\infty$, and all $L$

$$
\Leftrightarrow \bar{\sigma}\left[G K(I+G K)^{-1}\right]<1 / l_{m}(\omega)
$$

for all $0 \leqslant \omega<\infty$.

The last of these equations is the MIMO generalization of the familiar SISO requirement that loop gains be small whenever the magnitude of unstructured uncertainties is large. In fact, whenever $l_{m}(\omega) \gg 1$, we get the following constraint on $G K$ :

$$
\bar{\sigma}[G K(j \omega)]<1 / l_{m}(\omega)
$$

for all $\omega$ such that $l_{m}(\omega) \gg 1$.

We emphasize that these are not conservative stability conditions. On the contrary, if the uncertainties are truly unstructured and (17) is violated, then there exists a perturbation $L(s)$ within the set allowed by (13) for which the system is unstable. Hence, these stability conditions impose hard limits on the permissible loop gains of practical feedback systems.

\section{Performance Conditions}

Frequency domain conditions for requirement 3) have already been described in (5) in Section II. The only

${ }^{1}$ See any classical control text.

${ }^{2}$ If indentations are required, (14) and (17) must hold in the limit for all $\bar{s}$ on the indented path as the radius of indentation is taken to zero. 
modification needed to account for unstructured uncertainties is to apply (5) to $G^{\prime}$ instead of $G$, i.e.,

$$
\begin{aligned}
& p s \leqslant \underline{\sigma}[I+(I+L) G K] \\
& \Leftarrow p s \leqslant \underline{\sigma}\left[I+L G K(I+G K)^{-1}\right] \underline{\sigma}[I+G K] \\
& \Leftarrow \frac{p s(\omega)}{1-l_{m}(\omega)} \leqslant \underline{\sigma}[G K(j \omega)]
\end{aligned}
$$

for all $\omega$ such that $l_{m}(\omega)<1$ and $\sigma[G K(j \omega)] \gg 1$.

This is the MIMO generalization of another familiar SISO design rule-namely that performance objectives can be met in the face of unstructured uncertainties if the nominal loop gains are made sufficiently large to compensate for model variations. Note, however, that finite solutions exist only in the frequency range where $l_{m}(\omega)<1$.

The stability and performance conditions derived above illustrate that MIMO feedback design problems do not differ fundamentally from their SISO counterparts. In both cases, stability must be achieved nominally and assured for all perturbations by satisfying conditions (17) and (18). Performance may then be optimized by satisfying condition (19) as well as possible. What distinguishes MIMO from SISO design conditions are the functions used to express transfer function "size." Singular values replace absolute values. The underlying concepts remain the same.

We note that the singular value functions used in our statements of design conditions play a design role much like classical Bode plots. The $\bar{\sigma}[I+G K]$ function in (5) is the minimum return difference magnitude of the closedloop system, $\sigma[G K]$ in (8) and $\bar{\sigma}[G K]$ in (18) are minimum and maximum loop gains, and $\bar{\sigma}\left[G K(I+G K)^{-1}\right]$ in (17) is the maximum closed-loop frequency response. These can all be plotted as ordinary frequency dependent functions in order to display and analyze the features of a multivariable design. Such plots will here be called $\sigma$-plots.

One of the $\sigma$-plots which is particularly significant with regard to design for uncertainties is obtained by inverting condition (17), i.e.,

$$
l_{m}(\omega)<\frac{1}{\bar{\sigma}\left[G K(I+G K)^{-1}\right]}=\underline{\sigma}\left[I+G K(j \omega)^{-1}\right]
$$

for all $0 \leqslant \omega<\infty$.

The function on the right-hand side of this expression is an explicit measure of the degree of stability (or stability robustness) of the feedback system. Stability is guaranteed for all perturbations $L(s)$ whose maximum singular values fall below it. This can include gain or phase changes in individual output channels, simultaneous changes in several channels, and various other kinds of perturbations. In effect, $\sigma\left[I+(G K)^{-1}\right]$ is a reliable multivariable generalization of SISO stability margin concepts (e.g., frequency dependent gain and phase margins). Unlike the SISO case, however, it is important to note that $\underline{\sigma}[I+$ $(G K)^{-1}$ ] measures tolerances for uncertainties at the plant outputs only. Tolerances for uncertainties at the input are

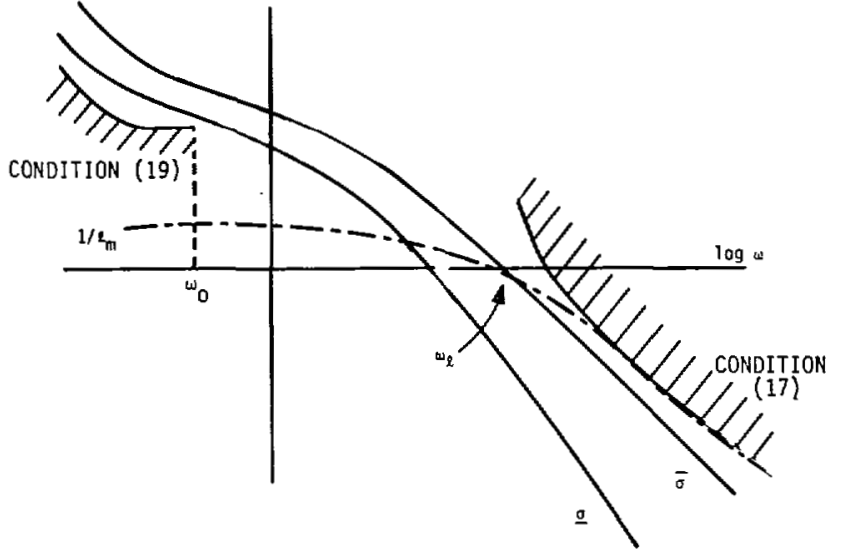

Fig. 3. The design tradeoff for $G K$.

generally not the same. They can be analyzed with equal ease, however, by using the function $\sigma\left[I+(K G)^{-1}\right]$ instead of $\sigma\left[I+(G K)^{-1}\right]$ in (20). This can be readily verified by evaluating the encirclement count of the map $\operatorname{det}(I+K G)$ under perturbations of the form $G^{\prime}=G(I+L)$ (i.e., uncertainties reflected to the input). The mathematical steps are directly analogous to (15)-(18) above.

Classical designers will recognize, of course, that the difference between these two stability robustness measures is simply that each uses a loop transfer function appropriate for the loop breaking point at which robustness is being tested.

\section{Transfer Function Limitations}

The feedback design conditions derived above are pictured graphically in Fig. 3. The designer must find a loop transfer function matrix, $G K$, for which the loop is nominally stable and whose maximum and minimum singular values clear the high and low frequency "design boundaries" given by conditions (17) and (19). The high frequency boundary is mandatory, while the low frequency one is desirable for good performance. Both are influenced by the uncertainty bound, $l_{m}(\omega)$.

The $\sigma$-plots of a representative loop transfer matrix are also sketched in the figure. As shown, the effective bandwidth of the loop cannot fall much beyond the frequency $\omega_{l}$ for which $l_{m}\left(\omega_{l}\right)=1$. As a result, the frequency range over which performance objectives can be met is explicitly constrained by the uncertainties. It is also evident from the sketch that the severity of this constraint depends on the rate at which $\sigma[G K]$ and $\bar{\sigma}[G K]$ are attenuated. The steeper these functions drop off, the wider the frequency range over which condition (19) can be satisfied. Unfortunately, however, FDLTI transfer functions behave in such a way that steep attenuation comes only at the expense of small $\underline{\sigma}[I+G K]$ values and small $\underline{\sigma}[I+$ $\left.(G K)^{-1}\right]$ values when $\sigma[G K]$ and $\bar{\sigma}[G K] \approx 1$. This means that while performance is good at lower frequencies and stability robustness is good at higher frequencies, both are poor near crossover. The behavior of FDLTI transfer 
functions, therefore, imposes a second major limitation on the achievable performance of feedback systems.

\section{SISO Transfer Function Limitation}

For SISO cases, the conflict between attenuation rates and loop quality at crossover is again well understood. We know that any rational, stable, proper, minimum phase loop transfer function satisfies fixed integral relations between its gain and phase components. Hence, its phase angle near crossover (i.e., at values of $\omega$ such that $|g k(j \omega)|$ $\approx 1$ ) is determined uniquely by the gain plot in Fig. 3 (for $\bar{\sigma}=\underline{\sigma}=|g k|)$. Various expressions for this angle were derived by Bode using contour integration around closed contours encompassing the right half plane $[5$, ch. 13, 14]. One expression is

$$
\begin{aligned}
\phi_{g k c} & \triangleq \arg \left[g k\left(j \omega_{c}\right)\right] \\
& =\frac{1}{\pi} \int_{-\infty}^{\infty} \frac{\ln |g k(j \omega(\nu))|-\ln \left|g k\left(j \omega_{c}\right)\right|}{\sinh \nu} d \nu
\end{aligned}
$$

where $\nu=\ln \left(\omega / \omega_{c}\right), \omega(\nu)=\omega_{c} \exp \nu$. Since the sign of $\sinh (\nu)$ is the same as the sign of $\nu$, it follows that $\phi_{g k c}$ will be large if the gain $|g k|$ attenuates slowly and small if it attenuates rapidly. In fact, $\phi_{g k c}$ is given explicitly in terms of weighted average attenuation rate by the following alternate form of (21) (also from [5]):

$$
\phi_{g k c}=\frac{1}{\pi} \int_{-\infty}^{\infty} \frac{d \ln |g k|}{d \nu}\left(\ln \operatorname{coth} \frac{|\nu|}{2}\right) d \nu .
$$

The behavior of $\phi_{g k c}$ is significant because it defines the magnitudes of our two SISO design conditions (17) and (19) at crossover. Specially, when $|g k|=1$, we have

$$
|1+g k| \equiv\left|1+(g k)^{-1}\right|=2\left|\sin \left(\frac{\pi+\phi_{g k c}}{2}\right)\right| .
$$

The quantity $\pi+\phi_{g k c}$ is the phase margin of the feedback system. Assuming $g k$ stable, this margin must be positive for nominal stability and, according to (23), it must be reasonably large $(\approx 1 \mathrm{rad})$ for good return difference and stability robustness properties. If $\pi+\phi_{g k c}$ is forced to be very small by rapid gain attenuation, the feedback system will amplify disturbances $(|1+g k| \ll 1)$ and exhibit little uncertainty tolerance at and near $\omega_{c}$. The conflict between attenuation rate and loop quality near crossover is thus clearly evident.

It is also known that more general nonminimum phase and/or unstable loop transfer functions do not alleviate this conflict. If the plant has right half-plane zeros, for example, it may be factored as

$$
g(s)=m(s) p(s)
$$

where $m(s)$ is minimum phase and $p(s)$ is an all-pass (i.e., $|p(j \omega)|=1 \quad \forall \omega$.$) The (negative) phase angle of p(s)$ reduces total phase at crossover, i.e.,

$$
\phi_{g k c}=\phi_{m k c}+\phi_{p c} \leqslant \phi_{m k c}
$$

and therefore aggravates the tradeoff problem. In fact, if $\left|\phi_{p c}\right|$ is too large, we will be forced to reduce the crossover frequency. Thus, RHP zeros limit loop gain (and thus performance) in a way similar to the unstructured uncertainty. A measure of severity of this added limitation is $|1-p(j \omega)|$, which can be used just like $l_{m}(\omega)$ to constrain a nominal minimum phase design.

If $g(s)$ has right half-plane poles, the extra phase lead contributed by these poles compared with their mirror images in the left half-plane is needed to provide encirclements for stability. Unstable plants thus also do not offer any inherent advantage over stable plants in alleviating the crossover conflict.

\section{Multivariable Generalization}

The above transfer limitations for SISO systems have multivariable generalizations; with some additional complications as would be expected. The major complication is that singular values of rational transfer matrices, viewed as functions of the complex variable $s$, are not analytic and therefore cannot be used for contour integration to derive relation such as (21). Eigenvalues of rational matrices, on the other hand, have the necessary mathematical properties. Unfortunately, they do not in general relate directly to the quality of the feedback design. (More is said about this in Section VI.) Thus, we must combine the properties of eigenvalues and singular values through the bounding relations

$$
\underline{\sigma}[A] \leqslant|\lambda[A]| \leqslant \bar{\sigma}[A]
$$

which holds for any eigenvalue, $\lambda_{i}$, of the (square) matrix $A$. The approach will be to derive gain/phase relations as in (21) for the eigenvalues of $I+G K$ and $I+(G K)^{-1}$ and to use these to bound their minimum singular values. Since good performance and stability robustness requires singular values of both of these matrices to be sufficiently large near crossover, the multivariable system's properties can then be no better than the properties of their eigenvalue bounds.

Equations for the eigenvalues themselves are straightforward. There is a one-to-one correspondence between eigenvalues of $G K$ and eigenvalues of $I+G K$ such that

$$
\lambda_{i}[I+G K]=1+\lambda_{i}[G K] .
$$

Likewise for $I+(G K)^{-1}$

$$
\lambda_{i}\left[I+(G K)^{-1}\right]=1+\frac{1}{\lambda_{i}[G K]} .
$$

Thus, when $\left|\lambda_{i}[G K]\right|=1$ for some $\lambda_{i}$ and $\omega=\omega_{c}$, we have

$$
\begin{aligned}
\left|\lambda_{i}[I+G K]\right| & \equiv\left|\lambda_{i}\left[I+(G K)^{-1}\right]\right| \\
& =2\left|\sin \left(\frac{\pi+\phi_{\lambda_{i} c}}{2}\right)\right| .
\end{aligned}
$$


Since this equation is exactly analogous to (23) for the scalar case, and since $\left|\lambda_{i}\right|$ bounds $\sigma$, it follows that the loop will exhibit poor properties whenever the phase angle $\left(\pi+\phi_{\lambda_{i} c}\right)$ is small.

In order to derive expressions for the angle $\phi_{\lambda_{1} c}$ itself, we require certain results from the theory of algebraic functions [20]-[26]. The key concepts needed from these references are that the eigenvalues $\lambda_{i}$ of a rational, proper transfer function matrix, viewed as a function of the complex variable $s$ constitute one mathematical entity, $\lambda(s)$, called an algebraic function. Each eigenvalue $\lambda_{i}$ is a branch of this function and is defined on one sheet of an extended Riemann surface domain. On its extended domain an algebraic function can be treated as an ordinary meromorphic function whose poles and zeros are the system poles and transmission zeros of the transfer function matrix. It also has additional critical points, called branch points, which correspond to multiple eigenvalues. Contour integration is valid on the Riemann surface domain provided that contours are properly closed.

In the contour integral leading to (21), $g k(s)$ may therefore be replaced by the algebraic function, $\lambda(s)$, with contour taken on its Riemann domain. Carrying out this integral yields several partial sums:

$$
\sum_{i} \phi_{\lambda, c}=\frac{1}{\pi} \int_{-\infty}^{\infty} \sum_{i} \frac{\left[\ln \left|\lambda_{i}(j \omega(\nu))\right|-\ln \left|\lambda_{i}\left(j \omega_{c}\right)\right|\right]}{\sinh \nu} d \nu
$$

where each sum is over all branches of $\lambda(s)$ whose sheets are connected by right half-plane branch points. Thus the eigenvalues $\left\{\lambda_{i}\right\}$ are restricted in a way similar to scalar transfer functions but in summation form. The summation, however, does not alter the fundamental tradeoff between attenuation rate and loop quality at crossover. In fact, if we deliberately choose to maximize the bound (29) by making $\omega_{c}$ and $\phi_{\lambda_{i} c}$ identical for all $i$, then (30) imposes the same restrictions on multivariable loops as (21) imposes on SISO loops. Hence, multivariable systems do not escape the fundamental transfer function limitations.

As in the scalar case, expression (30) is again valid for minimum phase systems only. That is, $G K$ can have no transmission zeros ${ }^{3}$ in the right half-plane. If this is not true, the tradeoffs governed by (29) and (30) are aggravated because every right half-plane transmission zero adds the same phase lag as in (25) to one of the partial sums in (30). The matrix $G K$ may also be factored, as in (24), to get

$$
G K(s)=M(s) P(s)
$$

where $M(s)$ has no right half-plane zeros and $P(s)$ is an all-pass matrix $P^{T}(-s) P(s)=I$. Analogous to the scalar case, $\bar{\sigma}(I-P(s))$ can be taken as a measure of the degree

${ }^{3}$ For our purposes, transmission zeros [41] are values $\bar{s}$ such that $\operatorname{det}[G(\bar{s}) K(\bar{s})]=0$. Degenerate systems with $\operatorname{det}[G K] \equiv 0$ for all $s$ are not of interest because they cannot meet condition (19) in Fig. 3. of multivariable nonminimum phaseness and used like $l_{m}(\omega)$ to constrain a nominal minimum phase design.

\section{Multivariable Design by Modern FREQUENCY DOMAIN METHODS}

So far, we have described the FDLTI feedback design problem as a design tradeoff involving performance objectives [condition (19)], stability requirements in the face of unstructured uncertainties [condition (17)], and certain performance limitations imposed by gain/phase relations which must be satisfied by realizable loop transfer functions. This tradeoff is essentially the same for SISO and MIMO problems. Design methods to carry it out, of course, are not.

For scalar design problems, a large body of welldeveloped tools exists (e.g., "classical control") which permits designers to construct good transfer functions for Fig. 3 with relatively little difficulty. Various attempts have been made to extend these methods to multivariable design problems. Probably the most successful of these are the inverse Nyquist array (INA) [3] and the characteristic loci (CL) methodologies [4]. Both are based on the idea of reducing the multivariable design problem to a sequence of scalar problems. This is done by constructing a set of scalar transfer functions which may be manipulated more or less independently with classical techniques. In the INA methodology, the scalar functions are the diagonal elements of a loop transfer function matrix which has been pre- and post-compensated to be diagonally dominant. In the $\mathrm{Cl}$ methodology, the functions are the eigenvalues of the loop transfer matrix.

Based on the design perspective developed in the previous sections, these multiple single-loop methods turn out to be reliable design tools only for special types of plants. Their restrictions are associated with the fact that the selected set of scalar design functions are not necessarily related to the system's actual feedback properties. That is, the feedback system may be designed so that the scalar functions have good feedback properties if interpreted as SISO systems, but the resulting multivariable system may still have poor feedback properties. This possibility is easy to demonstrate for the $\mathrm{CL}$ method and, by implication, for the INA method with perfect diagonalization. For these cases, we attempt to achieve stability robustness by satisfying

$$
l_{m}(\omega)<\left|\lambda_{i}\left[I+(G K)^{-1}\right]\right|=\left|1+1 / \lambda_{i}(G K)\right|
$$

for all $i$ and $0 \leqslant \omega<\infty$ and similarly, we attempt to achieve performance objectives by making

$$
\frac{p s(\omega)}{1-l_{m}(\omega)} \leqslant\left|\lambda_{i}[I+G K]\right|=\left|1+\lambda_{i}(G K)\right|
$$

for all $i$ and $0 \leqslant \omega<\infty$.

As discussed in Section V, however, the eigenvalues on the right-hand sides of these expressions are only upper 


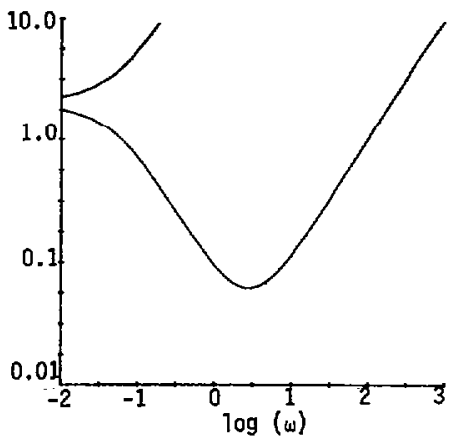

Fig. 4. $\sigma$-plots for $I+G^{-1}$. [ $G$ from (34).]

bounds for the true stability robustness and performance conditions (20) and (19). Hence, $\sigma\left[I+(G K)^{-1}\right]$ and/or $\sigma[I+G K]$ may actually be quite small even when (32) and (33) are satisfied.

\section{An Example}

These potential inadequacies in the INA and CL methods are readily illustrated with a simple example selected specifically to highlight the limitations. Consider

$$
G(s)=\frac{1}{(s+1)(s+2)}\left[\begin{array}{ll}
-47 s+2 & 56 s \\
-42 s & 50 s+2
\end{array}\right]
$$

This system may be diagonalized exactly by introducing constant compensation. Let

$$
U=\left[\begin{array}{ll}
7 & 8 \\
6 & 7
\end{array}\right] \quad U^{-1}=\left[\begin{array}{rr}
7 & -8 \\
-6 & 7
\end{array}\right]
$$

Then

$$
\hat{G}=U G U^{-1}=\left[\begin{array}{cc}
\frac{1}{s+1} & 0 \\
0 & \frac{2}{s+2}
\end{array}\right]
$$

If the diagonal elements of this $\hat{G}$ are interpreted as independent SISO systems, as in the INA approach, we could readily conclude that no further compensation is necessary to achieve desirable feedback properties. For example, unity feedback yields stability margins at crossover of $\pm \infty \mathrm{dB}$ in gain and greater than 90 degrees in phase. Thus, an INA design could reasonably stop at this point with compensator $K(s)=U U^{-1}=I$. Since the diagonal elements of $\hat{G}$ are also the eigenvalues of $G$, we could also be reasonably satisfied with this design from the $\mathrm{CL}$ point of view.

Singular value analysis, however, leads to an entirely different conclusion. The $\sigma$-plots for $\left(I+G^{-1}\right)$ are shown in Fig. 4. These clearly display a serious lack of robustness with respect to unstructured uncertainties. The smallest value of $\sigma$ is approximately 0.1 near $\omega=2 \mathrm{rad} / \mathrm{s}$. This means that multiplicative uncertainties as small as $l_{m}(2)=$ 0.1 ( $\approx 10 \%$ gain changes, $\approx 6$ deg phase changes) could produce instability. An interpretation of this lack of stabil-

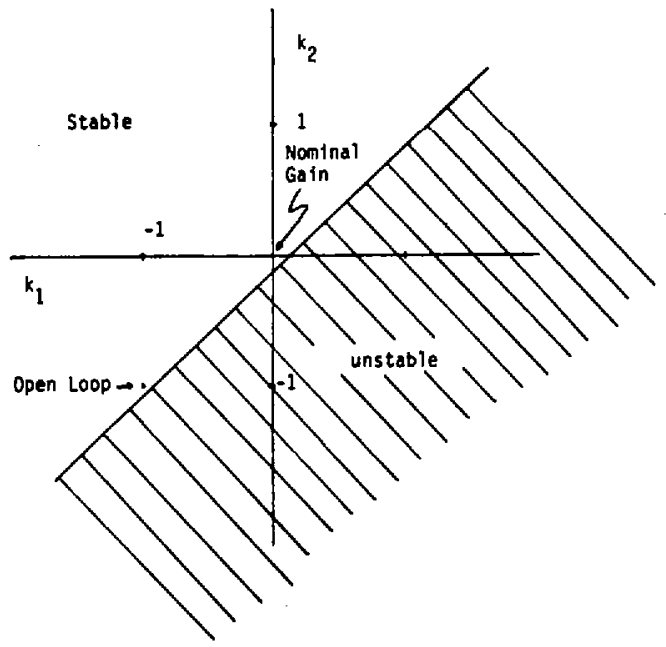

Fig. 5. Stability regions for $\left\{I+\left[\begin{array}{cc}k_{1} & 0 \\ 0 & k_{2}\end{array}\right]\right\} G$. [G from (34).]

ity robustness is given in Fig. 5. This figure shows stability regions in "gain space" for the compensator $K(s)=$ $\operatorname{diag}\left(1+k_{1}, 1+k_{2}\right)$. The figure reveals an unstable region in close proximity to the nominal design point. The INA and CL methods are not reliable design tools because they fail to alert the designer to its presence.

This example and the discussion which precedes it should not be misunderstood as a universal indictment of the INA and CL methods. Rather, it represents a caution regarding their use. There are various types of systems for which the methods prove effective and reliable. Conditions which these systems satisfy can be deduced from (32) and (33) - namely they must have tight singular value/eigenvalue bounds. This includes naturally diagonal systems, of course, and also the class of "normal" systems [28]. The limitations which arise when the bounds are not tight have also been recognized in [4].

We note in passing that the problem of reliability is not unique to the INA and CL methods. Various examples can be constructured to show that other design approaches such as the "single-loop-at-a-time" methods common in engineering practice and tridiagonalization approaches suffer similarly.

\section{Multivariable Design Via LQG}

A second major approach to multivariable feedback design is the modern LQG procedure [11], [12]. We have already introduced this method in connection with the tradeoff between command/disturbance error reduction and sensor noise error reduction. The method requires that we select stochastic models for sensor noise, commands and disturbances and define a weighted mean square error criterion as the standard of goodness for the design. The rest is automatic. We get an FDLTI compensator $K(s)$ which stabilizes the nominal model $G(s)$ (under mild assumptions) and optimizes the criterion of goodness. All too often, of course, the resulting loop 


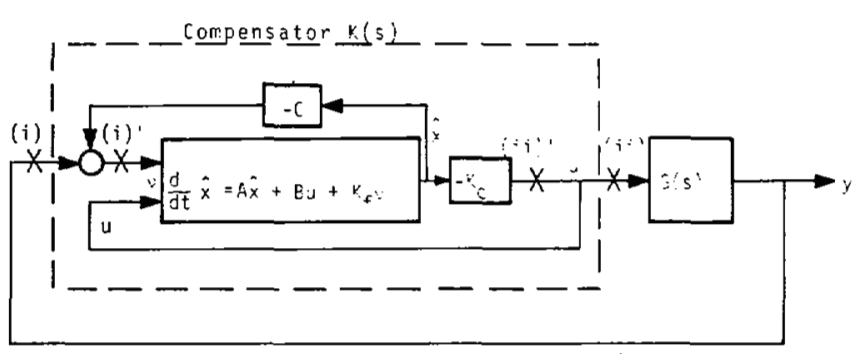

Fig. 6. LQG feedback loop.

transfer functions, $G K$ or $K G$, are entirely unacceptable when examined against the design constraints of Fig. 4. We are then forced to iterate the design-adjust weights in the performance criterion, change the stochastic disturbance and noise models, add dynamics, etc. There are so many parameters to manipulate that frustration sets in quickly and the schism between practitioners and theoreticians becomes easier to understand.

Fortunately, such design iterations of LQG controllers have become easier to carry out in the last few years because the frequency domain properties of these controllers are better understood. Some of the key new results are summarized below and their significance with respect to Fig. 3 are discussed. For our purposes, LQG controllers are ordinary FDLTI compensators with a special internal structure. This structure is shown in Fig. 6 and is well known. It consists of a Kalman-Bucy filter (KBF) designed for a state space realization of the nominal model $G(s)$, including all appended dynamics for disturbance processes, commands, integral action, etc. The model is

$$
\begin{aligned}
& \dot{x}=A x+B u+\xi ; \quad x \in R^{n}, \quad u \in R^{m} \\
& y=C x+\eta ; \quad y \in R^{r}
\end{aligned}
$$

and satisfies

$$
\begin{aligned}
G(s) & =C \Phi(s) B \\
d & =C \Phi(s) \xi
\end{aligned}
$$

with

$$
\Phi(s)=\left(s I_{n}-A\right)^{-1}
$$

The symbols $\xi$ and $\eta$ denote the usual white noise processes. The filter's gains are denoted by $K_{f}$ and its state estimates by $\hat{x}$. The state estimates are multiplied by full-state linear-quadratic regulator (LQR) gains, $K_{c}$, to produce the control commands which drive the plant and are also fed back internally to the KBF. The usual conditions for well-posedness of the LQG problem are assumed.

In terms of previous discussions, the functions of interest in Fig. 6 are the loop transfer, return difference, and stability robustness functions

$$
G K, \quad I_{r}+G K, \quad I_{r}+(G K)^{-1},
$$

and also their counterparts

$$
K G, \quad I_{m}+K G, \quad I_{m}+(K G)^{-1} .
$$

As noted earlier, the first three functions measure performance and stability robustness with respect to uncertainties at the plant outputs (loop-breaking point (i) in Fig. 6), and the second three measure performance and robustness with respect to uncertainties at the plant input (loopbreaking point (ii) in Fig. 6). Both points are generally significant in design.

Two other loop-breaking points, (i) ${ }^{\prime}$ and (ii)', are also shown in the figure. These are internal to the compensator and therefore have little direct significance. However, they have desirable loop transfer properties which can be related to the properties of points (i) and (ii). The properties and connections are these.

Fact 1: The loop transfer function obtained by breaking the LQG loop at point (i)' is the KBF loop transfer function $C \Phi K_{f}$.

Fact 2: The loop transfer function obtained by breaking the LQG loop at point ( $i$ ) is $G K$. It can be made to approach $C \Phi K_{f}$ pointwise in $s$ by designing the LQR in accordance with a "sensitivity recovery" procedure due to Kwakenaak [29].

Fact 3: The loop transfer function obtained by breaking the LQG loop at point (ii) ${ }^{\prime}$ is the LQR loop transfer function $K_{c} \Phi B$.

Fact 4: The loop transfer function obtained by breaking the LQG loop at point (ii) is $K G$. It can be made to approach $K_{c} \Phi B$ pointwise in $s$ by designing the KBF in accordance with a "robustness recovery" procedure due to Doyle and Stein [30].

Facts 1 and 3 can be readily verified by explicit evaluation of the transfer functions involved. Facts 2 and 4 take more elaboration and are taken up in a later section. They also require more assumptions. Specifically, $G(s)$ must be minimum phase with $m \geqslant r$ for Fact $2, m \leqslant r$ for Fact 4 , and hence, $G(s)$ must be square for both. Also, the names "sensitivity recovery" and "robustness recovery" are overly restrictive. "Full-state loop transfer recovery" is perhaps a better name for both procedures, with the distinction that one applies to points $(i),(i)^{\prime}$ and the other to points (ii), (ii)'.

The significance of these four facts is that we can design LQG loop transfer functions on a full-state feedback basis and then approximate them adequately with a recovery procedure. For point (i), the full state design must be done with the KBF design equations (i.e., its Riccati equation) and recovery with the LQR equations, while for point (ii), full-state design must be done with the LQR equations and recovery with the KBF. The mathematics of these two options are, in fact, dual. Hence, we will describe only one option [for point (ii)] in further detail. Results for the other are stated and used later in our example.

\section{Full-State Loop Transfer Design}

The intermediate full-state design step is worthwhile because LQR and KBF loops have good classical proper- 
ties which have been rediscovered over the last few years [31]-[33]. The basic result for the $L Q R$ case is that $L Q R$ loop transfer matrices

$$
T(s) \triangleq K_{c} \Phi(s) B
$$

satisfy the following return difference identity [32];

$$
\begin{aligned}
& {\left[I_{m}+T(j \omega)\right]^{*} R\left[I_{m}+T(j \omega)\right]} \\
& \quad=R+[H \Phi(j \omega) B]^{*}[H \Phi(j \omega) B] \quad \forall 0 \leqslant \omega<\infty
\end{aligned}
$$

where $R=R^{T}>0$ is the standard control weighting matrix, and $H^{T} H=Q \geqslant 0$ is the corresponding state weighting matrix. Without loss of generality, $H$ can be of size $(m \times n)$ [34]. Using the definitions (6) and (7), (41) with $R=\rho I$ implies that

$$
\begin{aligned}
\sigma_{i}\left[I_{m}+T(j \omega)\right] & =\sqrt{\lambda_{i}\left[I+\frac{1}{\rho}(H \Phi B)^{*} H \Phi B\right]} \\
& =\sqrt{1+\frac{1}{\rho} \lambda_{i}\left[(H \Phi B)^{*} H \Phi B\right]} \\
& =\sqrt{1+\frac{1}{\rho} \sigma_{i}^{2}[H \Phi(j \omega) B]} .
\end{aligned}
$$

This expression applies to all singular values $\sigma_{i}$ of $T(s)$ and, hence, specifically to $\sigma$ and $\bar{\sigma}$. It governs the performance and stability robustness properties of LQR loops.

\section{Performance Properties (Condition 19)}

Whenever $\sigma[T] \gg 1$, the following approximation of (42) shows explicitly how the parameters $\rho$ and $H$ influence $T(s)$ :

$$
\sigma_{i}[T(j \omega)] \approx \sigma_{i}[H \Phi(j \omega) B] / \sqrt{\rho} .
$$

We can thus choose $\rho$ and $H$ explicitly to satisfy condition (19) and also to "balance" the multivariable loop such that $\underline{\sigma}[T]$ and $\bar{\sigma}[T]$ are reasonably close together. ${ }^{4}$ This second objective is consistent with our assumption in Section III that the transfer function $G(s)$ has been scaled and/or transformed such at $l_{m}(\omega)$ applies more or less uniformly in all directions. This is also the justification for considering control weighting matrices in the form $R=\rho I$ only. Nonidentity $R$ 's are subsumed in $G$ as $G R^{1 / 2}$.

\section{Robustness Properties [Conditions (17) and (20)]}

It also follows from (42) that the LQR return difference always exceeds unity, i.e.,

$$
\underline{\boldsymbol{g}}\left[I_{m}+T(j \omega)\right] \geqslant 1 \quad \forall 0 \leqslant \omega<\infty .
$$

\footnotetext{
${ }^{4}$ It may also be necessary to append additional dynamics. In order to achieve zero steady state errors, for example, $\sigma[H \Phi B]$ must tend to $\infty$ as $\omega \rightarrow 0$. This may require additional integrations in the plant.
}

This implies [35] that

$$
\underline{\sigma}\left[I_{m}+T^{-1}(j \omega)\right] \geqslant 1 / 2 \quad \forall 0 \leqslant \omega<\infty .
$$

Hence, LQR loops are guaranteed to remain stable for all unstructured uncertainties (reflected to the input) which satisfy $l_{m}(\omega)<0.5$. Without further knowledge of the types of uncertainties present in the plant, this bound is the greatest robustness guarantee which can be ascribed to the regulator. ${ }^{5}$

While it is reassuring to have a guarantee at all, the $l_{m}<0.5$ bound is clearly inadequate for the requirements of condition (20) with realistic $l_{m}(\omega)^{\prime} s$. In order to satisfy condition (20) in LQR designs, therefore, it becomes necessary to directly manipulate the high-frequency behavior of $T(s)$. This behavior can be derived from known asymptotic properties of the regulator as the scalar $\rho$ tends to zero [29], [47], [48], [34], [36]. The result needed here is that under minimum phase assumptions on $H \Phi B$, the LQR gains $K_{c}$ behave asymptotically as [29]

$$
\sqrt{\rho} K_{c} \rightarrow W H
$$

where $W$ is an orthonormal matrix. The LQR loop transfer function, $T(s)$, evaluated at high frequencies, $s=j c / \sqrt{\rho}$ with $c$ constant, is then given by ${ }^{6}$

$$
\begin{aligned}
T(j c / \sqrt{\rho}) & =\sqrt{\rho} K_{c}(j c I-\sqrt{\rho} A)^{-1} B \\
& \rightarrow W H B / j c .
\end{aligned}
$$

Since crossovers occur at $\sigma_{i}[T]=1$, this means that the maximum (asymptotic) crossover frequency of the loop is

$$
\omega_{c \max }=\bar{\sigma}[H B] / \sqrt{\rho} .
$$

As shown in Fig 3, this frequency cannot fall much beyond $\omega_{l}$, where unstructured uncertainty magnitudes approach unity. Hence, our choice of $H$ and $\rho$ to achieve the performance objectives via (43) are constrained by the stability robustness requirement via (48).

Note also from (47) that the asymptotic loop transfer function in the vicinity of crossover is proportional to $1 / \omega$ ( -1 slope on log-log plots). This is a relatively slow attenuation rate which, in view of Section $V$, is the price the regulator pays for its excellent return difference properties. If $l_{m}^{-1}(\omega)$ attenuates faster than this rate, further reduction of $\omega_{c}$ may be required. It is also true, of course, that no physical system can actually maintain a $1 / \omega$ characteristic indefinitely [6]. This is not a concern here since $T(s)$ is a nominal (design) function only and will

\footnotetext{
${ }^{5}$ The $l_{m}<0.5$ bound turns out to be tight for pure gain changes, i.e., $0.5 \Leftrightarrow 6 \mathrm{~dB}$, which is identical to regulator's celebrated guaranteed gain margin [33]. The bound is conservative if the uncertainties are known to be pure phase changes, i.e., $0.5 \Leftrightarrow \approx 30 \mathrm{deg}$, which is less than the known \pm 60 deg guarantee [33].

${ }^{6} \mathrm{This}$ specific limiting process is appropriate for the so-called generic case [36] with full rank $H B$. More general versions of (55) with rank $[H B]<m$ are derived in [44].
} 
later be approximated by one of the full-state loop transfer recovery procedures.

\section{Full-State Loop Transfer Recovery}

As described earlier, the full-state loop transfer function designed above for point (ii) can be recovered at point (ii) by a modified $\mathrm{KBF}$ design procedure. The required assumptions are that $r \geqslant m$ and that $C \Phi B$ is minimum phase. The procedure then consists of two steps.

1) Append additional dummy columns to $B$ and zero row to $K_{c}$ to make $C \Phi B$ and $K_{c} \Phi B$ square $(r \times r)$. $C \Phi B$ must remain minimum phase.

2) Design the $\mathrm{KBF}$ with modified noise intensity matrices.

$$
\begin{aligned}
& E\left(\xi \xi^{T}\right)=\left[M_{0}+q^{2} B B^{T}\right] \delta(t-\tau) \\
& E\left(\eta \eta^{T}\right)=N_{0} \delta(t-\tau)
\end{aligned}
$$

where $M_{0}, N_{0}$ are the nominal noise intensity matrices obtained from stochastic models of the plant and $q$ is a scalar parameter. Under these conditions, it is known that the filter gains $K_{f}$ have the following asymptotic behavior as $q \rightarrow \infty$ [30]:

$$
\frac{1}{q} K_{f} \rightarrow B W N_{0}^{-1 / 2}
$$

Here $W$ is another orthonormal matrix, as in (46). When this $K_{f}$ is used in the loop transfer expression for point (ii), we get pointwise loop transfer recovery as $q \rightarrow \infty$, i.e.,

$$
\begin{aligned}
K(s) G(s)= & K_{c}\left[\Phi^{-1}+B K_{c}+K_{f} C\right]^{-1} K_{f} C \Phi B \\
= & K_{c}\left[\bar{\Phi}-\bar{\Phi} K_{f}\left(I_{r}+C \bar{\Phi} K_{f}\right)^{-1} C \bar{\Phi}\right] K_{f} C \Phi B \\
= & K_{c} \bar{\Phi} K_{f}\left(I_{r}+C \bar{\Phi} K_{f}\right)^{-1} C \Phi B \\
\rightarrow & K_{c} \bar{\Phi} B(C \bar{\Phi} B)^{-1} C \Phi B \\
= & K_{c} \Phi B\left(I_{r}+K_{c} \Phi B\right)^{-1} \\
& \cdot\left[C \Phi B\left(I_{r}+K_{c} \Phi B\right)^{-1}\right]^{-1} C \Phi B \\
= & \left\{K_{c} \Phi B(C \Phi B)^{-1}\right\} C \Phi B \\
= & K_{c} \Phi B
\end{aligned}
$$

In this series of expressions, $\bar{\Phi}$ was used to represent the matrix $\left(s I_{n}-A+B K_{c}\right)^{-1}$, (49) was used to get from (52) to (53), and the identity $\bar{\Phi} B=\Phi B\left(I+K_{c} \Phi B\right)^{-1}$ was used to get from there to (54). The final step shows explicitly that the asymptotic compensator $K(s)$ [the bracketed term in (55)] inverts the nominal plant (from the left) and substitutes the desired LQR dynamics. The need for minimum phase is thus clear, and it is also evident that the entire recovery procedure is only appropriate as long as the target LQR dynamics satisfy Fig. 3's constraints (i.e., as long as we do not attempt inversion in frequency ranges where uncertainties do not permit it). Closer inspection of (50)-(56) further shows that there is no dependence on LQR or KBF optimality of the gains $K_{c}$ or $K_{f}$. The procedure requires only that $K_{f}$ be stabilizing and have the asymptotic characteristic (49). Thus, more general state feedback laws can be recovered (e.g., pole placement), and more general filters can be used for the process (e.g., observers) ${ }^{7}$

\section{An Example}

The behavior of LQG design iterations with full-state loop transfer recovery is illustrated by the following abstracted longitudinal control design example for a $\mathrm{CH}-47$ tandem rotor helicopter. Our objective is to control two measured outputs-vertical velocity and pitch attitudeby manipulating collective and differential collective rotor thrust commands. A nominal model for the dynamics relating these variables at 40 knot airspeed is [45]

$$
\begin{aligned}
\frac{d}{d t} x= & {\left[\begin{array}{clrc}
-0.02 & 0.005 & 2.4 & -32 \\
-0.14 & 0.44 & -1.3 & -30 \\
0 & 0.018 & -1.6 & 1.2 \\
0 & 0 & 1 & 0
\end{array}\right] x } \\
& +\left[\begin{array}{cc}
0.14 & -0.12 \\
0.36 & -8.6 \\
0.35 & 0.009 \\
0 & 0
\end{array}\right] u \\
y= & {\left[\begin{array}{llll}
0 & 1 & 0 & 0 \\
0 & 0 & 0 & 57.3
\end{array}\right] x . }
\end{aligned}
$$

Major unstructured uncertainties associated with this model are due to neglected rotor dynamics and unmodeled rate limit nonlinearities. These are discussed at greater length in [46]. For our present purposes, it, suffices to note that they are uniform in both control channels and that $l_{m}(\omega)>1$ for all $\omega \geqslant 10 \mathrm{rad} / \mathrm{s}$. Hence, the controller bandwidth should be constrained as in Fig. 3 to $\omega_{c \max } \leqslant 10$.

Since our objective is to control two measured outputs at point (i), the design iterations utilize the duals of (40)-(56). They begin with a full state KBF design whose noise intensity matrices, $E\left(\xi \xi^{T}\right)=\Gamma \Gamma^{T} \delta(t-\tau)$ and $E\left(\eta \eta^{T}\right)$ $=\rho I \delta(t-\tau)$, are selected to meet performance objectives at low frequencies, i.e.,

$$
\underline{\sigma}[T] \approx \underline{\sigma}[C \Phi \Gamma] / \sqrt{\rho} \geqslant p s,
$$

while satisfying stability robustness constraints at high frequencies,

$$
\omega_{c \max }=\bar{\sigma}[C \Gamma] / \sqrt{\rho}<10 \mathrm{r} / \mathrm{s} .
$$

7Still more generally, the modified KBF procedure will actually recover full-state feedback loop transfer functions at any point, $u^{l}$, in the system for which $C \Phi B^{1}$ is minimum phase [30]. 


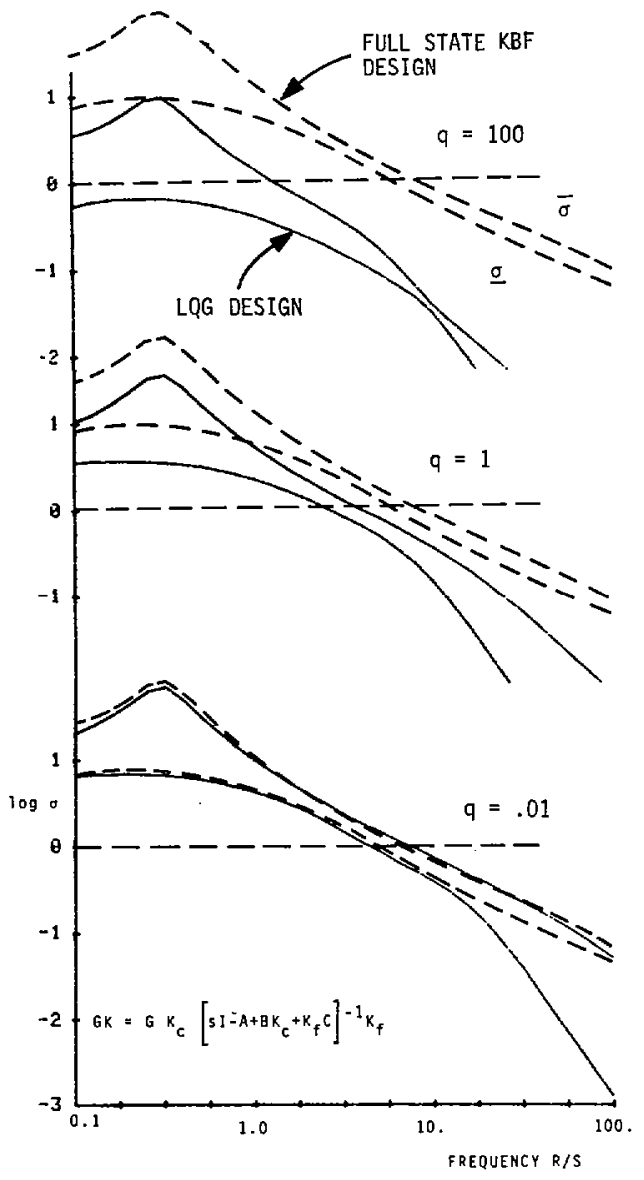

Fig. 7. Full-state loop transfer recovery.

For the choice $\Gamma=B,(58)$ constrains $\rho$ to be greater than or equal to unity. ${ }^{8}$ The resulting KBF loop transfer for $\rho=1$ is shown in Fig. 7. For purposes of illustration, this function will be considered to have the desired high gain properties for condition (19), with low gains beyond $\omega=10$ for condition (20). ${ }^{9}$ It then remains to recover this function by means of the full-state recovery procedure for point (i). This calls for LQR design with $Q=Q_{0}+q^{2} C C^{T}$ and $R=R_{0}$. Letting $Q_{0}=0, R_{0}=I$, the resulting LQG transfer functions for several values of $q$ are also shown in Fig. 7. They clearly display the pointwise convergence properties of the procedure.

\section{CONClusion}

This paper has attempted to present a practical design perspective on MIMO linear time invariant feedback control problems. It has focused on the fundamental issuefeedback in the face of uncertainties. It has shown how

\footnotetext{
${ }^{8}$ If $C \Gamma$ (or $H B$ ) is singular, (58) or (48) are still valid in the nonzero directions.

${ }^{9}$ The function should not be considered final, or course. Better balance between $\bar{\sigma}$ and $\sigma$ and greater gain at low frequencies via appended integrators would be desirable in a serious design.
}

classical SISO approaches to this issue can be reliably generalized to MIMO systems, and has defined the extent to which MIMO systems are subject to the same uncertainty constraints and transfer function gain/phase limitations as SISO ones. Two categories of design procedures, were then examined in the context of these results.

There are numerous other topics and many other proposed design procedures which were not addressed, of course. Modal control, [42] eigenvalue-vector assignments, [43] and the entire field of geometric methods [19] are prime examples. These deal with internal structural properties of systems which, though important theoretically, cease to have central importance in the face of the inputoutput nature of unstructured incertainties. Hence, they were omitted. We also did not treat certain performance objectives in MIMO systems which are distinct from SISO systems. These include perfect noninteraction and integrity. Noninteraction is again a structural property which loses meaning in the face of unstructured uncertainties. [It is achieved as well as possible by condition (19).] Integrity, on the other hand, cannot be dismissed as lightly. It concerns the ability of MIMO systems to maintain stability in the face of actuator and/or sensor failures. The singular value concepts described here are indeed useful for integrity analysis. For example, a design has integrity with respect to actuator failures whenever

$$
\underline{\sigma}\left[I+(K G)^{-1}\right]>1 \quad \forall \omega .
$$

This follows because failures satisfy $l_{m} \leqslant 1$. Moreover it can be shown [37] that full-state control laws designed via Lyapunov equations, as opposed to Riccati equations, as in Section VII, satisfy (59). It is also worth noting that integrity properties claimed for design methods such as INA and CL suffer from the reliability problem discussed in Section VI and, hence, may not be valid in the system's natural (nondiagonal) coordinate system.

The major limitations on what has been said in the paper are associated with the representation chosen in Section III for unstructured uncertainty. A single magnitude bound on matrix perturbations is a worst case representation which is often much too conservative (i.e., it may admit perturbations which are structurally known not to occur). The use of weighted norms in (8) and (9) or selective transformations applied to $G$ (as in [39]) can alleviate this conservatism somewhat, but seldom completely. For this reason, the problem of representing more structured uncertainties in simple ways analogous to (13) is receiving renewed research attention [38].

A second major drawback is our implicit assumption that all loops (all directions) of the MIMO system should have equal bandwidth ( $\sigma$ close to $\bar{\sigma}$ in Fig. 3). This assumption is consistent with a uniform uncertainty bound but will no longer be appropriate as we learn to represent more complex uncertainty structures. Research along these lines is also proceeding. 


\section{REFERENCES}

[1] M. Athans and P. L. Falk, Optimal Control. New York: McGraw-Hill, 1966.

[2] A. E. Bryson and Y. C. Ho, Applied Optimal Control. Waltham, MA: Ginn, 1969.

[3] H. H. Rosenbrock, Computer-Aided Control System Design. New York: Academic, 1974.

[4] A. G. J. MacFarlane and B. Kouvaritakis, "A design technique for linear multivariable feedback systems," Int. J. Contr., vol. 25 , pp. 837-879, 1977.

[5] H. W. Bode, Network Analysis and Feedback Amplifier Design. Princeton, NJ: Van Nostrand, 1945.

[6] I. M. Horowitz, Synthesis of Feedback Systems. New York: Academic, 1963.

[7] J. B. Cruz, Ed., System Sensitivity Analysis. Stroudsburg, PA: Dowden, Hutchinson \& Ross, 1973.

[8] B. S. Garbow et al., Matrix Eigensystem Routines-EISPACK Guide Extension, Lecture Notes in Computer Science, vol. 51. New York: Springer-Verlag, 1977.

[9] G. W. Stewart, Introduction to Matrix Computations. New York: Academic, 1973.

[10] I. M. Horowitz and U. Shaked, "Superiority of transfer function over state variable methods," IEEE Trans. Automat. Contr., pp. 84-97, Feb. 1975.

[11] Special Issue on the LQG Problems, IEEE Trans. Automat. Contr., Dec. 1971.

[12] D. C. Youla, H. A. Jabr, and J. J. Bongiorno, Jr., "Modern Wiener-Hopf design of optimal controllers-Part II, The multivariable case," IEEE Trans. Automat. Contr., June 1976.

[13] G. Stein, G. L. Hartmann, and R. C. Hendrick, "Adaptive control laws for F-8C flight test," IEEE Trans. Automat. Contr., Oct. 1977.

[14] M. G. Safonov, "Robustness and stability aspects of stochastic multivariable feedback system design," Ph.D. dissertation, Massachusetts Institute of Technology, 1977.

[15] G. Zames, "On the input-output stability of time-varying nonlinear feedback systems-Part I," IEEE Trans. Automat. Contr., vol. AC-11, no. 2, pp. 228-238, Apr. 1966.

"On the input-output stability of time-varying nonlinear feedback systems - Part II," IEEE Trans. Automat. Contr., vol. AC-11, no. 3 pp. 465-476, July 1966.

[16] R. L. Bisplinhoff, H. Ashley and R. L. Halfman, Aeroelasticity. Reading, MA: Addison-Wesley, 1957.

[17] C. E. J. Bowler, D. N. Ewart, and C. Concordia, "Self-excited torsional frequency oscillations with series capacitors," IEEE Trans. Power App. Syst., Sept. /Oct. 1973.

[18] H. H. Rosenbrock, "The stability of multivariable systems," IEEE Trans. Automat. Contr., vol. AC-17, pp. 105-107, Feb. 1972.

[19] W. M. Wonham, Linear Multivariable Control: A Geometric Approach. New York: Springer-Verlag, 1974.

[20] K. Knopp, Theory of Functions, vol. II. New York: Dover, 1947.

[21] G. Springer, Introduction to Riemann Surfaces. Reading, MA: Addison-Wesley, 1957.

[22] G. A. Bliss, Algebraic Functions. New York: Dover, 1966 (reprint of 1933 original).

[23] A. G. J. MacFarlane and I. Postlethwaite, "Characteristic frequency functions and characteristic gain functions," $I J C$, vol. 26, pp. $265-278,1977$.

[24] A. G. J. MacFarlane, and I. Postlethwaite, "The generalized Nyquist stability criterion and multivariable root loci," IJC, vol. 25 , pp. 81-127, 1977.

[25] A. G. J. MacFarlane, B. Kouvaritakis, and J. M. Edmunds, "Complex variable methods for multivariable feedback systems analysis and design," Alternatives for Linear Multivariable Control, National Engineering Consortium, Chicago, IL, pp. 189-228, 1977.

[26] I. Postlethwaite, and A. G. J. MacFarlane, $A$ Complex Variable Approach to the Analysis of Linear Multivariable Feedback Systems. Berlin: Springer-Verlag, 1979.

[27] C. A. Desoer, and M. Vidyasagar Feedback Systems: Input-Output Properties. New York: Academic, 1975.

[28] Gantmacher, The Theory of Matrices. New York: Chelsea, 1960.

[29] H. Kwakernaak and R. Sivan, Linear Optimal Control Systems. New York: Wiley-Interscience, 1972.

[30] J. C. Doyle and G. Stein, "Robustness with observers," IEEE Trans. Automat. Contr., Aug. 1979.

[31] R. E. Kalman, "When is a linear system optimal?" Trans. ASME Ser. D: J. Basic Eng., vol. 86, pp. 51-60, 1964.

[32] B. D. O. Anderson and J. B. Moore, Linear Optimal Control. Englewood Cliffs, NJ: Prentice-Hall, 1971.

[33] M. G. Safonov and M. Athans, "Gain and phase margin of multiloop LQG regulators," IEEE Trans. Automat. Contr., Apr. 1977 .

[34] C. A. Harvey and G. Stein, "Quadratic weights for asymptotic regulator properties," IEEE Trans. Automat. Contr., vol. AC-23, pp. $378-387,1978$.

[35] A. J. Laub, "Robust stability of linear systems-Some computational considerations," Laboratory for Information and Decision Systems, M.I.T., LIDS-R-904, Feb. 1979.

[36] G. Stein, "Generalized quadratic weights for asymptotic regulator properties," IEEE Trans. Automat. Contr., vol. AC-24, pp. 559-566, 1979.

[37] C. A. Harvey, "On feedback systems possessing integrity with respect to actuator outages," in Proc. MIT/ONR Workshop on Recent Developments in the Robustness Theory of Multivariable Systems, MIT Rep. LIDS-R-954, Apr. 1979.

[38] M. F. Barrett, "Conservatism with sector-based robustness tests," Ph.D. dissertation, Univ. of Minnesota, 1980.

[39] M. G. Safonov, "Frequency-domain design of multivariable control systems for insensitivity to large plant modeling errors," presented at the18th CDC, Fort Lauderdale, FL, Dec. 12-14, 1979.

[40] H. H. Rosenbrock, "Design of multivariable control systems using the inverse Nyquist array," Proc. IEEE, vol. 110, no. 11, Nov. 1969.

[41] A. G. J. MacFarlane and N. Karcanias, "Poles and zeros of linear multivariable systems: A survey of algebraic, geometric, and complex variable theory," Int. J. Contr., pp. 33-74, July 1976.

[42] J. D. Simon and S. K. Mitter, "A theory of modal control," Inform. Contr., vol. 13, pp. 316-353, 1968.

[43] B. C. Moore, "On the flexibility offered by state feedback in multivariable systems beyond closed loop eigenvalue assignment," IEEE Trans. Automat. Contr., vol. AC-21, pp. 685-692, 1976.

[44] G. Stein, "Asymptotic loop transfer functions of LQ regulators and filters," in preparation.

[45] G. L. Hartmann, G. Stein, and S. G. Prath, "Digital adaptive controllers for VTOL vehicles," NASA Contractor Rep. 159 NASA Langley Research Center, Nov. 1979.

[46] G. Stein and J. C. Doyle, "Singular values and feedback: Design examples," in Proc. 1978 Allerton Conf., Urbana, IL, 1978.

[47] Shaked, "The asymptotic behavior of the root loci of multivariable optimal regulators," IEEE Trans. Automat. Contr., pp. 425-430, June 1978.

[48] B. Kouvaritakis, "The optimal root loci of linear multivariable systems," Int. J. Contr., pp. 33-62, July 1978.

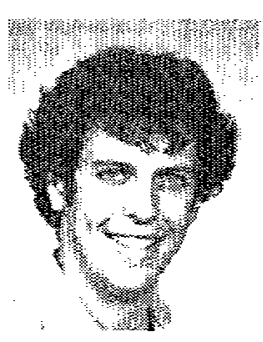

John C. Doyle received the S.B. and S.M. degrees in electrical engineering from the Massachusetts Institute of Technology in 1977.

He now divides his time between consulting work for Honeywell Systems and Research Center in Minneapolis, MN and graduate studies in mathematics at the University of California at Berkeley. His research interest in the area of automatic control is in developing theory that is relevant to the practical problems faced by engineers.

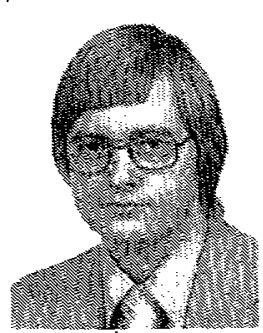

Gunter Stein (S'66-M'69) received the B.S.E.E. degree from General Motors Institute, Flint, MI, and the M.S.E.E. and Ph.D. degrees from Purdue University, West Lafayette, IN, in 1966 and 1969, respectively.

Since 1969 he has been with the Honeywell Systems and Research Center, Minneapolis, MN. As of January 1977, he also has held an Adjunct Professor appointment with the Department of Electrical Engineering and Computer Sciences, Massachusetts Institute of Technology, Cambridge. His research interests are to make modern control theoretical methods work.

Dr. Stein is a member of AIAA, Eta Kappa Nu, and Sigma Xi. He has served as Chairman of the Technical Committee on Applications (19741975), IEEE Transactions on Automatic Control, and as Chairman of the 14th Symposium on Adaptive Processes. 